\title{
\begin{tabular}{|l|l|l|l|l}
\hline M & R & S & Internet Journal of & Nitride Semiconductor Research \\
\hline
\end{tabular}
}

Volume 2, Article 20

\section{Evidence of 2D-3D transition during the first stages of GaN growth on AIN}

\author{
F. Widmann, B. Daudin, G. Feuillet, Y. Samson, M. Arlery, J. L. Rouviere \\ CEA/Grenoble, Département de Recherche Fondamentale sur la Matière Condensée/SP2M
}

This article was received on July 8, 1997 and accepted on August 21, 1997.

\begin{abstract}
In order to identify the strain relaxation mechanism, Molecular Beam Epitaxy of wurtzite GaN on AIN was monitored in situ using Reflection High Energy Electron Diffraction (RHEED). In the substrate temperature range between $620^{\circ} \mathrm{C}$ and $720^{\circ} \mathrm{C}$, a Stransky-Krastanov (SK) transition was evidenced, resulting in a 2D-3D transition after completion of 2 monolayers, with subsequent coalescence of 3D islands, eventually resulting in a smooth surface. Quantitative analysis of the RHEED pattern allowed us to determine that island formation is associated with elastic relaxation. After island coalescence, a progressive plastic relaxation is observed. The size and density of 3D islands was varied as a function of the growth parameters. AFM experiments revealed that the size of the GaN islands, about $8 \mathrm{~nm}$ large and $2 \mathrm{~nm}$ high, was small enough to expect quantum effects. It was found that capping of the islands by AIN resulted in a smooth surface after deposition of a few monolayers allowing us to grow a "superlattice" of islands by periodically repeating the process.
\end{abstract}

\section{Introduction}

In order to grow high quality heterostructures of nitride semiconductors, such as GaN/AIN/GaN SIS structures, or GaN/AIN superlattices and superalloys, it is important to control the strain relaxation mechanism of GaN on AIN, and conversely of AIN on GaN, for which the mismatch is $2.5 \%$. Until now studies dealing with this problem have been scarce. For example, Bykhovski et al. [1] calculated a critical thickness, but to the best of our knowledge, the mechanism of strain relaxation and its kinetics has never been studied in detail. In particular, the point of whether the relaxation process is totally plastic or also elastic needs to be clarified.

Elastic relaxation through 3D islanding, or Stransky Krastanov (SK) growth mode, is commonly observed in a large variety of systems, the most widely studied being InAs/GaAs [2]. GaN islands formation has already been reported by Tanaka et. al. [3], but in this case the growth, that was undertaken by CVD, was not controlled by an intrinsic SK mode, and the size of the dots was rather large (typically $40 \mathrm{~nm}$ ).

Self-organized and controlled growth of quantum dots is of primary interest in the case of nitrides. In particular, the luminescence properties of laser diodes are believed to originate from InGaN quantum dots [4], and controlled formation of such structures would be a way to improve the properties of the devices.

In this article, we study the strain relaxation process of GaN grown on AIN by Molecular Beam Epitaxy, using in situ RHEED analysis, and we demonstrate that the formation of GaN dots on AIN can be controlled by a careful adjustment of the growth parameters.

\section{Experimental}

The growth is carried out using a commercial Meca 2000 Molecular Beam Epitaxy system, equipped with Al and $\mathrm{Ga}$ effusion cells. Active nitrogen is produced by a EPI - Unibulb RF plasma source. The (0001) sapphire plane is used for the growth of GaN and AIN. The substrates are degreased in a standard way, and cleaned using a hot mixture of $\mathrm{H}_{2} \mathrm{SO}_{4}: \mathrm{H}_{3} \mathrm{PO}_{4} / 3: 1$ during 10 minutes. They are then heated at $750^{\circ} \mathrm{C}$ in the deposition chamber, and exposed during 90 minutes to the nitrogen plasma. From the observation of the 
RHEED pattern during this nitridation step, it is believed that the $\mathrm{Al}_{2} \mathrm{O}_{3}$ surface is transformed into a thin layer with an in-plane lattice parameter about $3 \%$ smaller than that of AIN [5]. On this nitrided $\mathrm{Al}_{2} \mathrm{O}_{3}$ surface, a $250 \AA$ thick AIN buffer layer is grown at a temperature of $550^{\circ} \mathrm{C}$. The temperature is then raised to $720{ }^{\circ} \mathrm{C}$ for further growth of the AIN layer. This nucleation process allows for the formation of a very smooth AIN surface, immediately after the deposition of the first monolayers. The AIN thickness is about $5000 \AA$, which is high enough for the layer to be fully relaxed.

The growth of GaN on the AIN layer is followed by RHEED. The RHEED pattern is recorded during the whole growth process using a videotape recorder. The intensity profiles of the $(10)$ and $\left(\begin{array}{ll}\overline{1} & 0\end{array}\right)$ streaks are then fitted with gaussian curves and their spacing is computed with a high precision. This method allows direct in situ measurement of the in-plane lattice parameter variations, with a precision better than $0.2 \%$. In addition, the RHEED pattern gives information on the roughness of the growth front, which can be followed as a function of the deposition time. This is done by measuring the intensity of a Bragg diffraction spot and by comparing it to the intensity of the streak, away from the spot. The variation of both in-plane lattice parameter and roughness are then determined as a function of time. RHEED intensity oscillations obtained during GaN growth on previous samples allowed us to calibrate the growth rate according to the experimental conditions [6].

\section{Results}

Before starting GaN growth, the AIN surface is exposed to the nitrogen flux, at the GaN growth temperature $\left(680^{\circ} \mathrm{C}\right)$. It exhibits a smooth RHEED pattern, with intense and thin streaks. A two-fold reconstruction is observable, which has been assigned to a nitrogen-stabilized surface [7].

During the first 10 seconds of growth, no diffraction spots are observed : the growth remains two-dimensional ( Figure 1). After 10 seconds, a transition occurs : the roughness increases abruptly, and at the same time the lattice parameter rises towards a value closer to that of relaxed GaN. This behavior is assigned to island formation, following the completion of two monolayers critical thickness, corresponding to the first of 10 seconds of the growth. Before the transition, the growing GaN layer is strained and two-dimensional. After the transition, the strain is relaxed elastically through the free surface of the islands. This relaxation is not complete, since the variation is only $1.5 \%$ instead of $2.5 \%$, as would be expected if GaN were totally relaxed on AIN. The size of the island might be of importance for whether all or only part of the strain is relaxed.

This process of elastic strain relaxation through 3D islanding after completion of a thin 2D wetting layer is typical of a Stransky-Krastanov growth mode, as already observed in other systems [2] [8].

After island formation, one can observe that the roughness, as well as the lattice parameter, decreases. This is assigned to the coalescence of the islands, which smoothes the surface. The free surface of the islands is reduced during their coalescence, and therefore the layer is strained again, leading to a decrease of the in-plane lattice parameter.

For the reverse case of AIN grown on GaN, no elastic relaxation has been observed, and the growth remains two-dimensional from the beginning.

The GaN relaxation process during the first stages of growth is temperature dependent. At high temperature, the 2D-3D transition occurs, but after the islands coalescence, the growth remains 3-dimensional. At lower temperature, the transition is almost absent, and the layer remains 2-dimensional and strained. More details about the effect of temperature on the 2D-3D transition are given in [9].

To study the islands, the growth was stopped just after the 2D-3D transition, at the roughness maximum. The growth temperature was $710{ }^{\circ} \mathrm{C}$, and the equivalent of about 4 monolayers of GaN were deposited. The dots obtained have been observed using Atomic Force Microscopy (Figure 2). The density was very high $\left(6.10^{11} \mathrm{~cm}\right.$ -2 ), and dots were estimated around $7 \mathrm{~nm}$ wide. and 1.8 to $2 \mathrm{~nm}$ high, taking into account the radius of curvature of the tip apex.

To change the size and the density of the islands, dots grown in the same conditions as above have been exposed to nitrogen plasma during one minute just after their growth. The density, about $5.10^{10} \mathrm{~cm}^{-2}$, is lower than in the previous case (Figure 3). The size is also bigger (around $25 \mathrm{~nm}$ wide and $4.5 \mathrm{~nm}$ high), which suggests that during the exposure to an active nitrogen flux, the larger dots grow to the expense of the smaller ones. The size of the dots could thus be controlled by changing the exposure time under nitrogen plasma, but can also be varied with the substrate temperature and the amount of deposited GaN. 
As the dots and the tip apex exhibit similar radius, AFM is not very precise for determining the shape of the dots. In the RHEED pattern of the dots along the $\langle 11 \overline{2} \quad 0>$ azimuth, one can see well defined facets, with an angle of $30^{\circ}$ relative to the surface (Figure 4 ). When rotating the sample, these facets appear to have a six-fold symmetry. From these observations, and the dimensions of the dots determined by AFM (diameter and height), one can deduce that the dots are truncated pyramids, with an hexagonal basis (Figure 5).

Transmission Electron Microscopy (TEM) has been carried out on a sample which consisted in a stacking of 3 layers of GaN dots, separated with 160 A thick AIN spacer layer (Figure 6). The growth conditions correspond to the ones of the small dots observed by AFM. A careful analysis of High Resolution Electron Microsopy images allows us to measure the thickness of the two-dimensional GaN wetting layer. We find that the wetting thickness varies slightly from 1 to 3 monolayers, which is in good agreement with RHEED measurements. The interfaces between the AIN layers and the GaN wetting monolayers are flat, which confirms that the growth of AIN on GaN is two-dimensional, and that it is possible to smooth the surface by capping the islands with AIN. The sizes of the islands observed by TEM are in agreement to the ones deduced from AFM.

\section{Conclusion}

It was demonstrated, using quantitative RHEED analysis, that the strain of a GaN layer grown on AIN is relaxed through a Stransky-Krastanov growth mode, with 3D islanding occurring after a critical thickness of 2 monolayers. After this elastic relaxation that occurs during the first stages of growth, it is believed that a slower plastic relaxation takes place.

Using this SK behavior, GaN dots of a nanometric size where obtained, from which quantum confinement effects are expected [9]. They have been characterized using AFM and TEM. The size and the density of the dots can be controlled by precise adjustment of the growth parameters : substrate temperature, deposition and nitridation times.

Further work will concern the photoluminescence and cathodoluminescence of the GaN dots. Calculations have to be performed in order to correlate their optical and morphological properties.

\section{References}

[1] A. D. Bykhovski , B. L. Gelmont, M. S. Shur , J. Appl. Phys. 78, 3691-3696 (1995).

[2] J. M. Moison, F. Houzay, F. Barthe, L. Leprince, E. Andre, O. Vatel, Appl. Phys. Lett. 64, 196 (1994).

[3] S Tanaka, S Iwai, Y Aoyagi, Appl. Phys. Lett. 69, 4096-4098 (1996).

[4] Y. Narukawa, Y. Kawakami, M. Funato, S. Fujita, S. Fujita, S. Nakamura, Appl. Phys. Lett. 70, 981-983 (1997).

[5] B Daudin, F Widmann, unpublished (1997).

[6] G Feuillet, F Widmann, B Daudin, J Schuler, M Arlery, JL Rouviere, N Pelekanos, O Briot, unpublished (1997).

[7] P Hacke, G Feuillet, H Okumura, S Yoshida, Appl. Phys. Lett. 69, 2507-2509 (1996).

[8] D. J. Eaglesham, M. Cerullo, Phys. Rev. Lett. 64, 1943 (1990).

[9] B Daudin, F Widmann, G Feuillet, C Adelmann, Y Samson, M Arlery, JL Rouviere, unpublished (1997). 


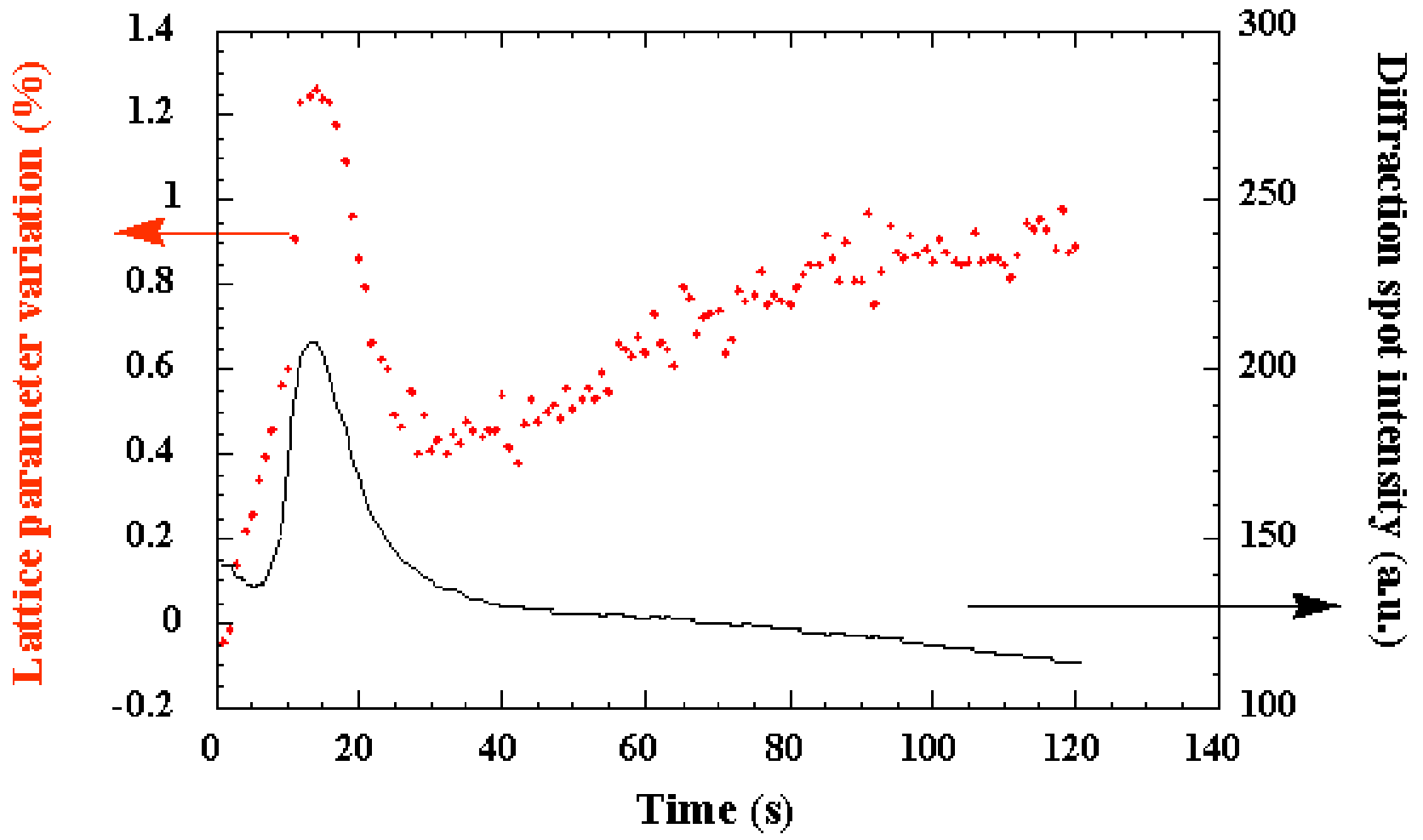

Figure 1. Variation, as a function of time, of the in-plane lattice parameter deduced from the spacing between the RHEED streaks (red dots) and of the 3D character of the growth, deduced from the intensity of a Bragg diffraction spot (black line). Growth rate is $0.25 \mathrm{ML} / \mathrm{s}$.

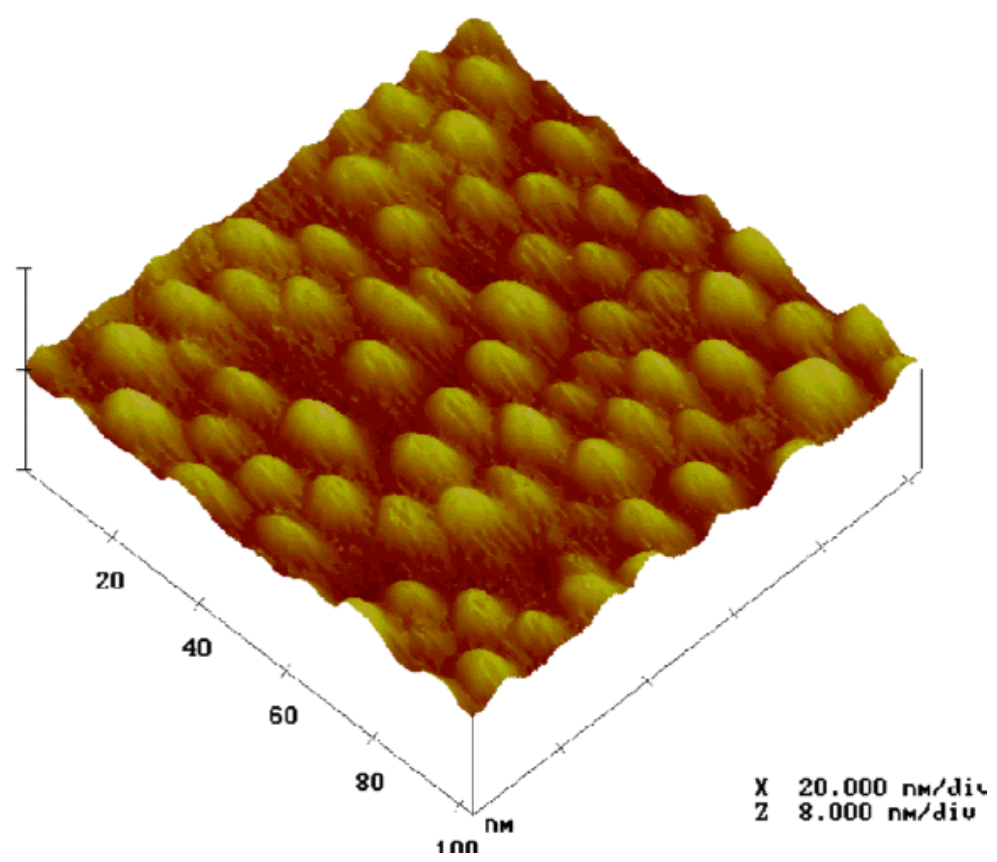

Figure 2. AFM Image (tapping mode) of GaN dots grown on AIN, at a temperature of $710^{\circ} \mathrm{C}$, without post-growth reorganization under nitrogen plasma. 


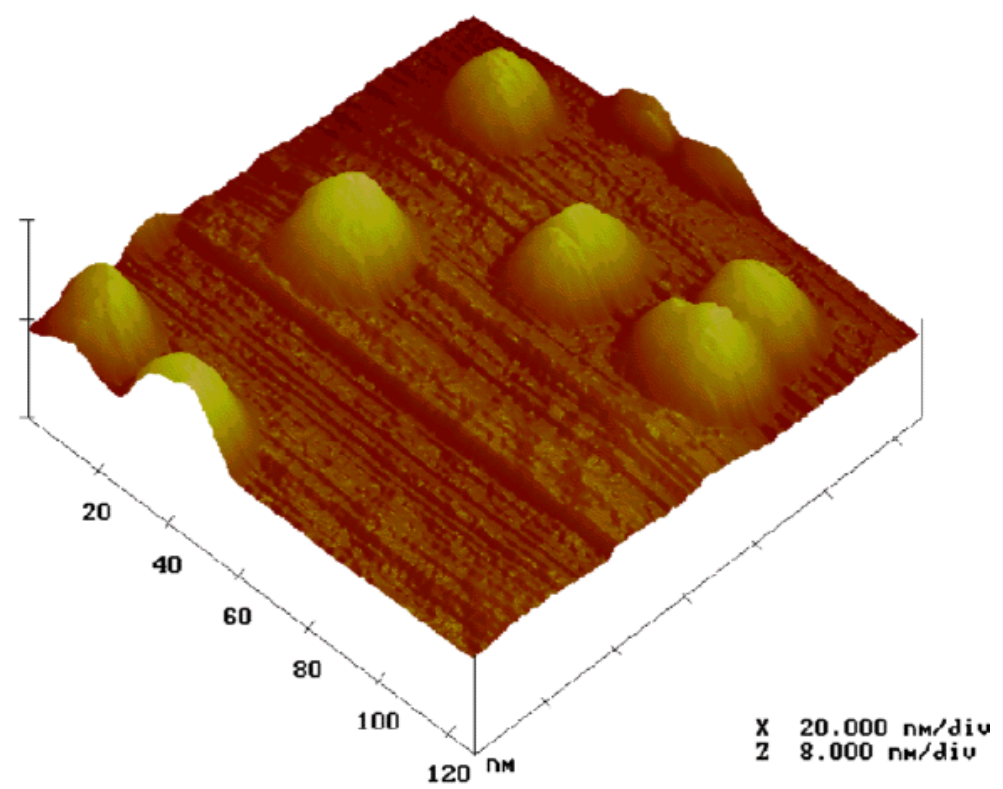

Figure 3. AFM image (tapping mode) of GaN dots grown on AIN at $710^{\circ} \mathrm{C}$, and exposed during $50 \mathrm{~s}$ to nitrogen plasma after growth.

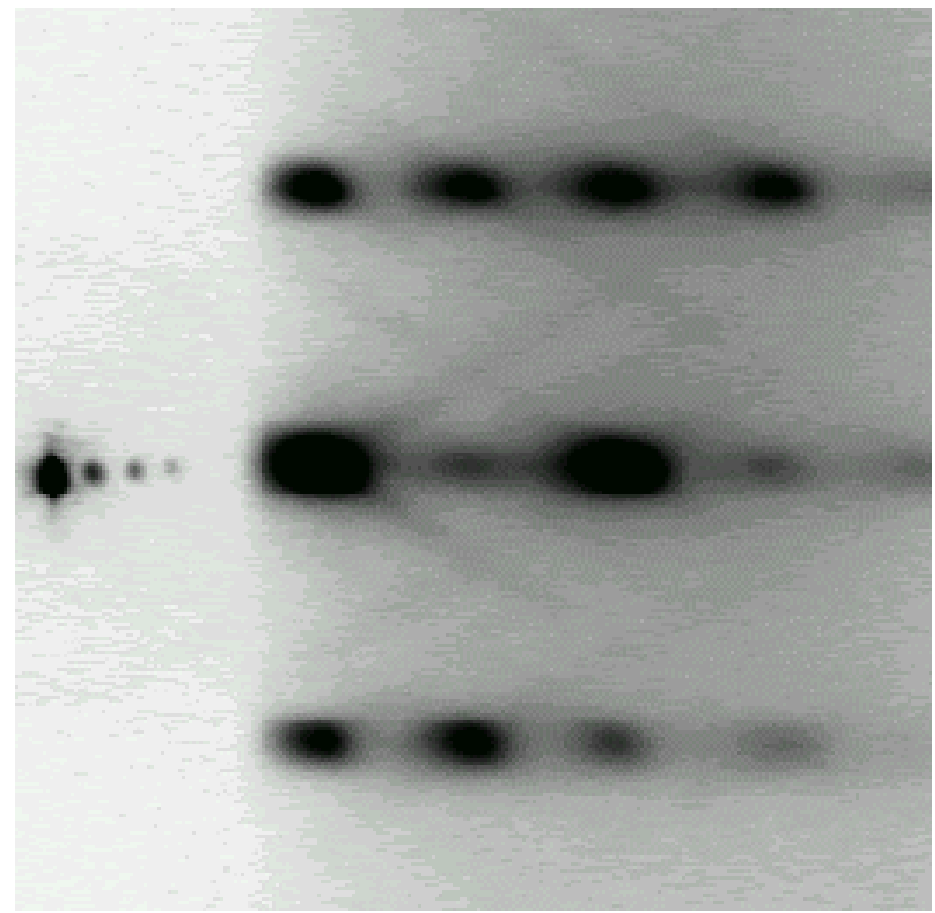

Figure 4. RHEED pattern of GaN dots after exposure to nitrogen flux, revealing the presence of facets at $30^{\circ}$ from the surface. The electron beam is along the $<11 \overline{2} \quad 0>$ azimuth. 


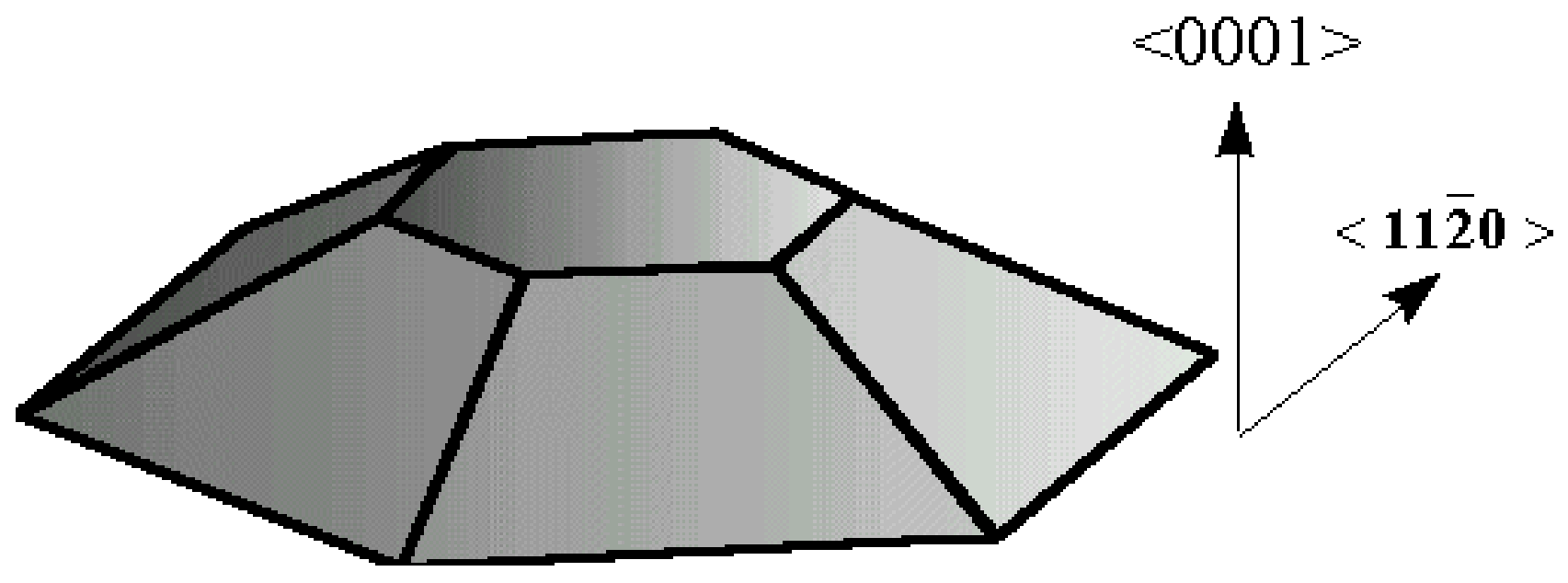

Figure 5. Schematic view of a GaN island, deduced from RHEED and AFM observations.

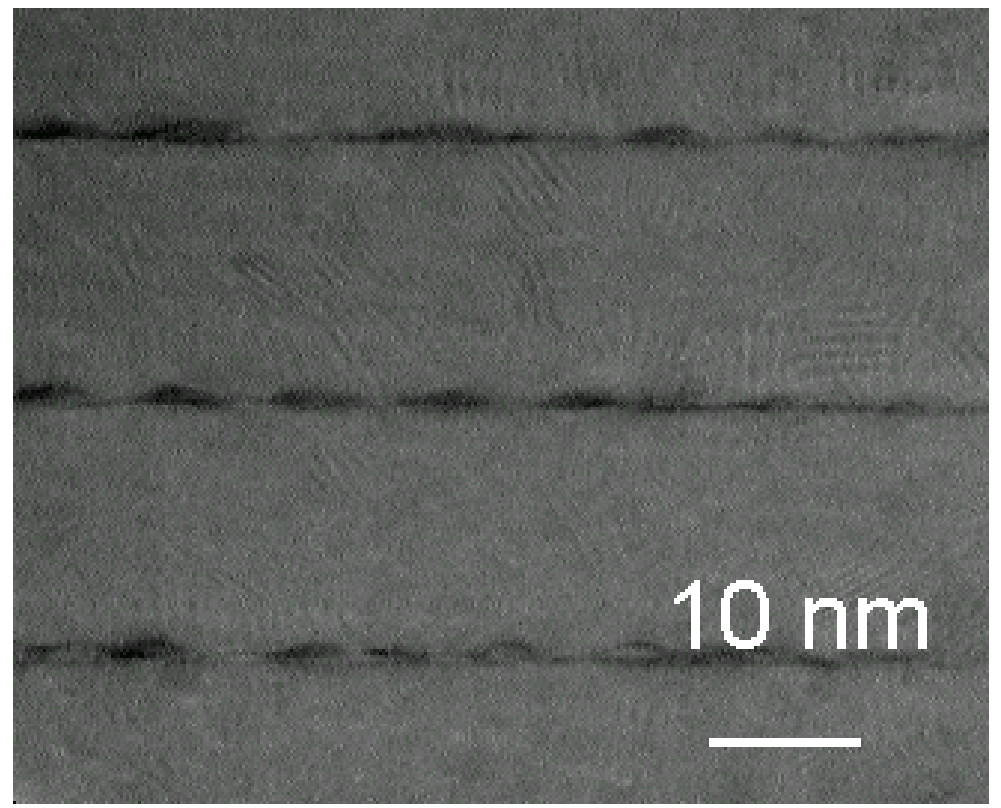

Figure 6. TEM image of GaN dots embedded in AIN. The dots have been grown at $710^{\circ} \mathrm{C}$, by depositing the equivalent of 4 monolayers of GaN, and have been capped with AIN immediately after their growth.

(C) 1997 The Materials Research Society

\begin{tabular}{|l|l|l|l|l|}
\hline M & $R$ & $S$ & Internet Journal of & Nitride Semiconductor Research \\
\hline
\end{tabular}

